\title{
Using GANs with adaptive training data to search for new molecules
}

\author{
Andrew E. Blanchard (0, Christopher Stanley and Debsindhu Bhowmik*
}

\begin{abstract}
The process of drug discovery involves a search over the space of all possible chemical compounds. Generative Adversarial Networks (GANs) provide a valuable tool towards exploring chemical space and optimizing known compounds for a desired functionality. Standard approaches to training GANs, however, can result in mode collapse, in which the generator primarily produces samples closely related to a small subset of the training data. In contrast, the search for novel compounds necessitates exploration beyond the original data. Here, we present an approach to training GANs that promotes incremental exploration and limits the impacts of mode collapse using concepts from Genetic Algorithms. In our approach, valid samples from the generator are used to replace samples from the training data. We consider both random and guided selection along with recombination during replacement. By tracking the number of novel compounds produced during training, we show that updates to the training data drastically outperform the traditional approach, increasing potential applications for GANs in drug discovery.
\end{abstract}

Keywords: Generative Adversarial Network, Drug discovery, Search

\section{Introduction}

From materials design to drug discovery, many scientific endeavors with significant practical applications can be viewed as a search over the space of all possible chemical compounds [1,2]. Due to the high-dimensional nature of the search space, an exhaustive enumeration of possible candidates is not feasible [1]. To overcome this difficulty, traditional approaches in drug discovery have relied upon domain knowledge from physics and chemistry to construct synthesis rules to guide the search for new compounds. However, reliance on current knowledge to generate rules may unnecessarily limit the amount of chemical space explored [2].

In recent years, a data driven approach has emerged to empower searches over chemical space. Deep learning models have been constructed to learn lower dimensional representations of data to identify meaningful clusters and discover related compounds with a desired

*Correspondence: bhowmikd@ornl.gov

Computational Sciences and Engineering Division, Oak Ridge National Laboratory, Oak Ridge, TN 37830, USA functionality [3-7]. Of particular interest to drug discovery, machine learning (ML) models have been incorporated into pipelines for iterative refinement of candidates. More specifically, generative models have been utilized as a key component for providing novel molecules for targeted experimental investigations $[1,2,8]$.

Generative models in machine learning seek to recreate the distribution underlying a given set of data. After modeling the distribution, new samples can be drawn that extend the original data. One type of generative approach, known as a Generative Adversarial Network (GAN), has been widely used in many applications from image generation to drug discovery [9-12]. Recent studies have utilized GANs to search the space of possible molecules for drug design, developing models that can generate compounds with a desired feature set $[11,12]$.

Although generative models (and GANs) have many advantages for finding new molecules, a key limitation is the propensity for mode collapse $[8,13]$. In mode collapse, the model distribution collapses to cover only a few samples from the training data. Beyond mode collapse, it is intuitively expected that a given 

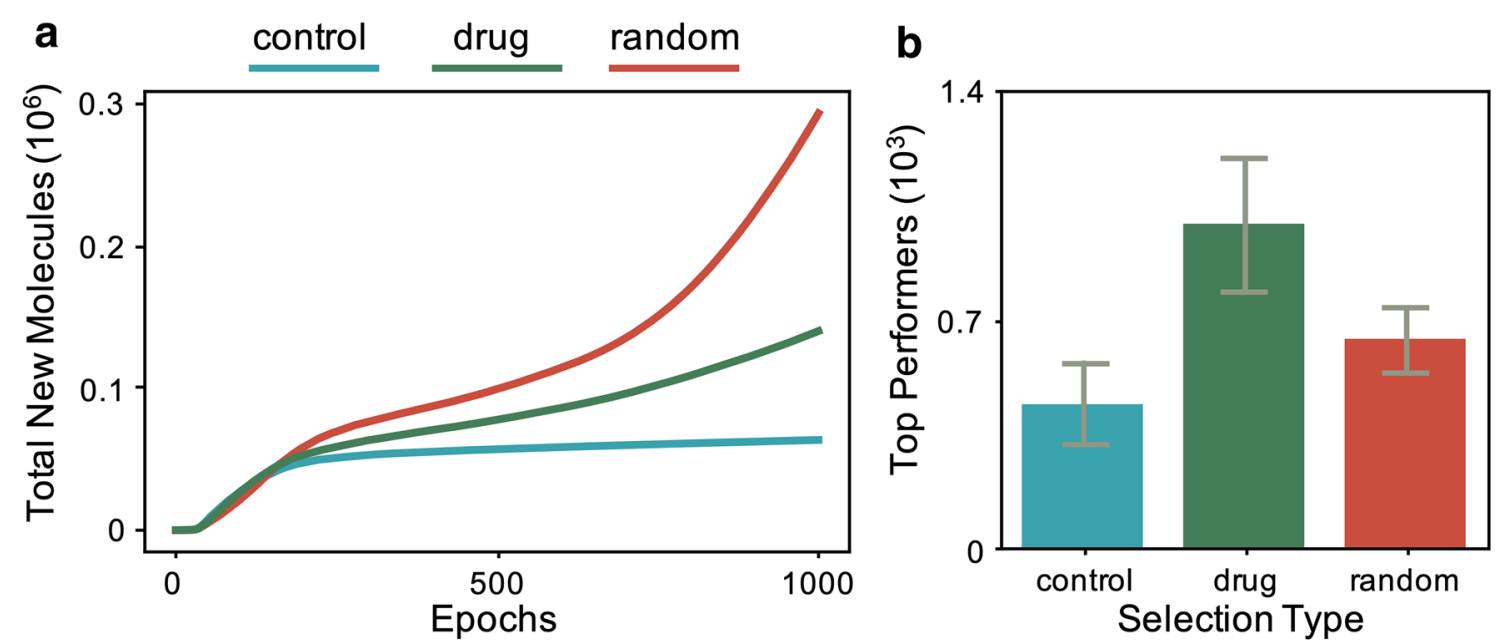

Fig. 1 New molecules produced for different replacement strategies. For control (blue), the training data is fixed. For random (red), molecules from the generator randomly replace molecules in the training data. For drug (green), molecules from the generator only replace training samples if they have a higher drug-likeness score. a As training progresses, control stops producing a substantial number of new molecules, but random and drug replacement strategies continue production. Plot shows average over three training runs for each selection type. b Although drug produces less overall new molecules than random, it generates more top performers. Plot shows average over three runs for each selection type with error bars showing one standard deviation

generative model will be limited by the training data used (i.e. there is no standard way to guide the generative model in areas of parameter space that it has never encountered in training). This limitation hinders the use of GANs in search applications such as drug discovery. To overcome mode collapse, several approaches have been investigated including updating the loss function to promote diversity $[12,14,15]$. However, these approaches rely on comparisons to a fixed training data set, which continues to hinder search applications.

Here, we build upon recent work utilizing GANs for small molecule discovery [11] by introducing a new approach for training. Our approach enables augmented search through incremental updating of the training data set using ideas from Genetic Algorithms [16]. Novel and valid molecules that are generated by the model are stored during a training interval. Then, the training data is updated through a replacement strategy, which can be guided or random. Training resumes and the process is repeated. Our results show that this approach can alleviate the decrease in new molecules generated that occurs for a standard GAN during training. Furthermore, we utilize recombination between generated molecules and the training data to increase new molecule discovery. Introducing replacement and recombination into the training process empowers the use of GANs for broader searches in drug discovery.

\section{Results and discussion}

To improve the search capabilities of GANs, we updated the training process to include concepts from Evolutionary (e.g. Genetic) Algorithms [16]. For a typical Genetic Algorithm, a parent population is used to generate a child population through mutation and recombination. The parent population is then updated (i.e. replaced) using selection based on specified fitness criteria. For our purposes, the training data is the population under consideration. The generator from the GAN produces candidates for the child population over multiple training epochs, and recombination occurs between the new candidates and the parent generation. Through replacement, the training data adapts to better reflect new areas explored by the generator.

As a first step in using adaptive training data for GANs, we consider replacement without recombination on a training set from QM9 [17]. In this case, we have three different types of training: control, random, drug. For control, the training data is held fixed while the GAN is trained. For random, the training data is updated by the generated molecules. For drug, the training data is updated only by generated molecules that outperform the current samples on quantitative estimation of druglikeness score (i.e. drug-likeness) $[18,19]$

As shown in Fig. 1a, the control GAN produces new molecules during the initial stage of training, but quickly reaches a plateau. Intuitively this is expected, as the generator learns to mimic the training data, the 

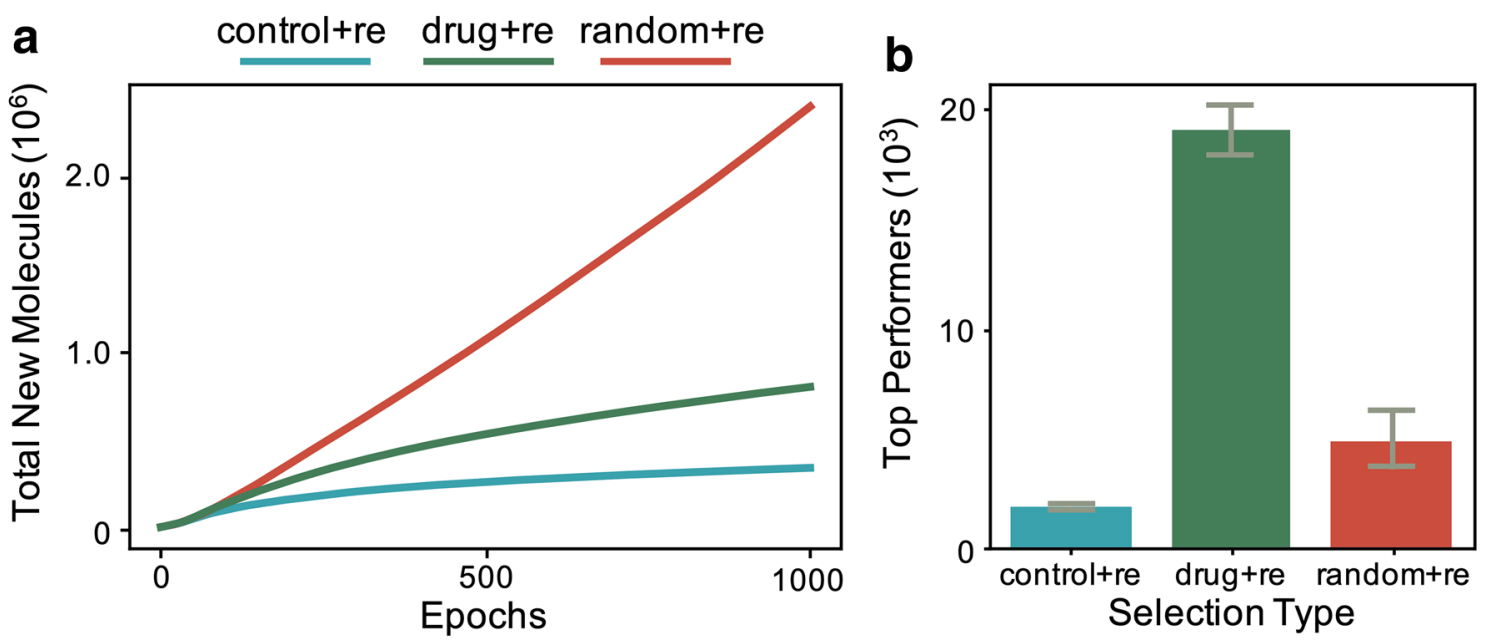

Fig. 2 New molecules produced for different replacement strategies with recombination. For control+re (blue), the training data is fixed. For random+re (red), molecules from the generator randomly replace molecules in the training data. For drug+re (green), molecules from the generator only replace training samples if they have a higher drug-likeness score. a Similar to the case without recombination, random and drug replacement strategies outperform control as training progresses. Plot shows average over three training runs for each selection type. $\mathbf{b}$ Although drug+re produces less overall new molecules than random+re, it generates more top performers. Plot shows average over three runs for each selection type with error bars showing one standard deviation

number of novel molecules produced decreases. Alternatively, for random and drug replacement, the GAN continues to produce new molecules over the entire training period as the training data is updated.

Although the number of new molecules produced is an important metric for drug discovery, when optimizing for some feature (e.g. drug-likeness), the quality of the generated samples is also key. As shown in Fig. 1b, drug replacement is able to generate the most top performers even though it generates fewer new molecules than random. Here, we define top performers as having a drug-likeness score above a threshold of 0.6, corresponding to the approximate mean value of optimized molecules in previous work [11, 12]. Similar results are shown for additional metrics (i.e. synthesizability and solubility) in Additional file 1: Figures S1, S2. Notice that the metric-specific selection strategy generates the most top performers for each metric considered.

In addition to selection/replacement, another common mechanism in Genetic Algorithms to introduce diversity into a population is recombination. We included recombination into our approach by taking half of the generated molecules and applying crossover with a sample from the current training data. As shown in Fig. 2a, the same hierarchy as the case without recombination is observed. Recombination, however, does increase the absolute number of new molecules produced drastically. The increase in new molecules also translates to many more high performers (Fig. $2 \mathrm{~b}$. Similar results are shown for additional metrics (i.e. synthesizability and solubility) in Additional file 1: Figures S3, S4.

Beyond the bulk performance metrics shown in Fig. 2, Fig. 3 shows specific examples of top performers

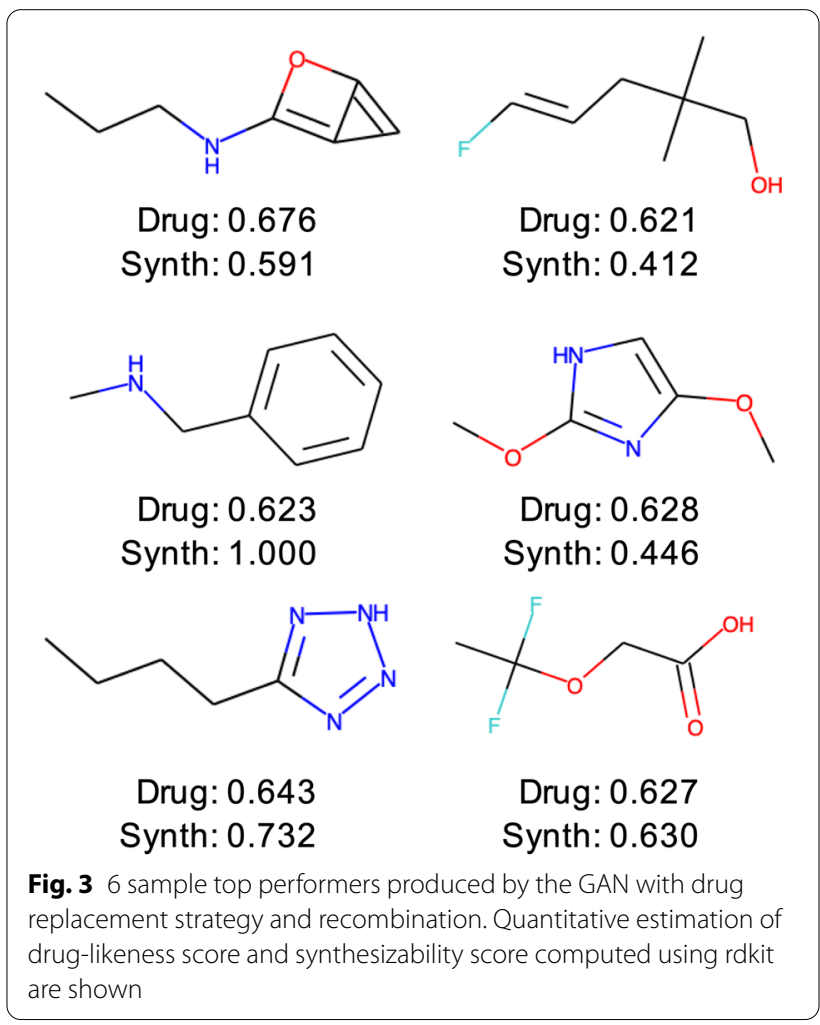



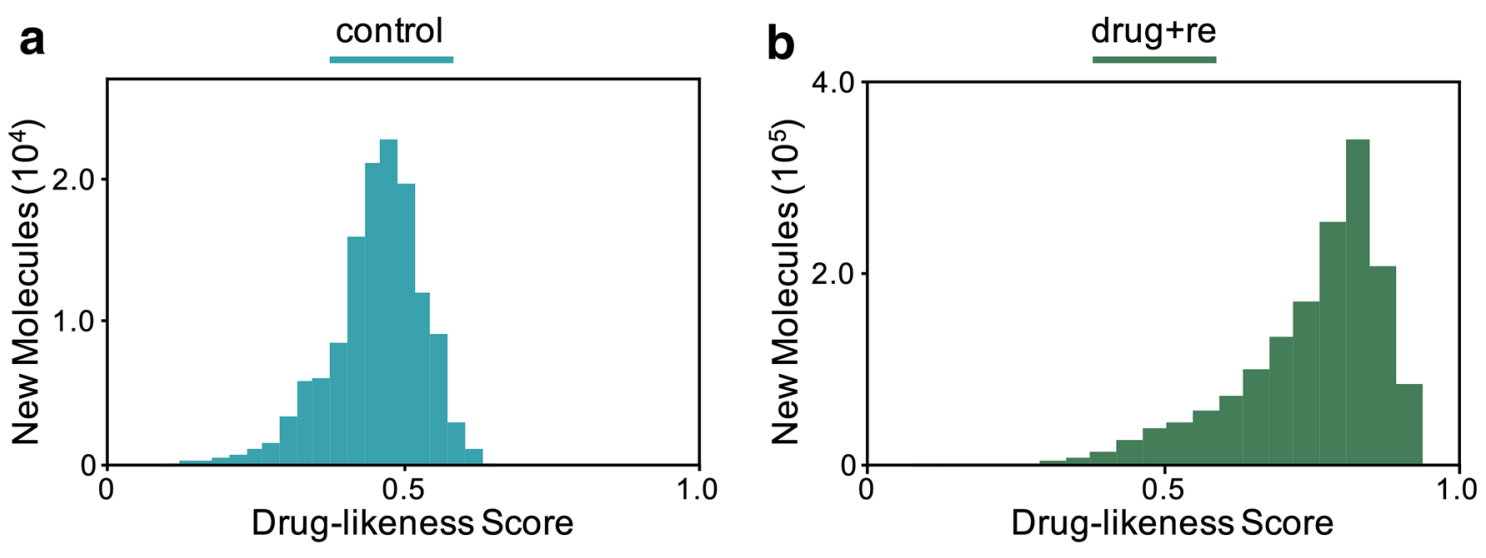

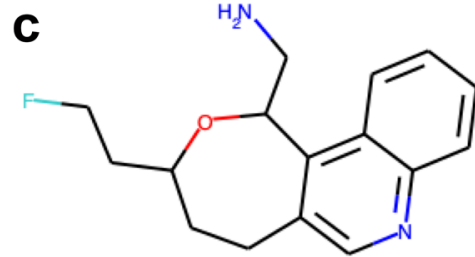

Drug: 0.936

Synth: 0.400

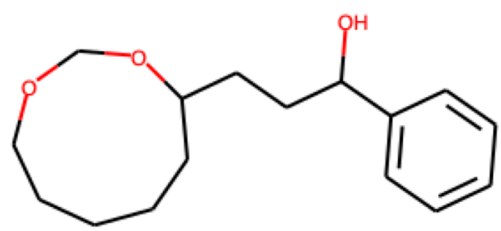

Drug: 0.905

Synth: 0.477<smiles>CN(C)c1ccccc1ON1CCNc2ccccc21</smiles>

Drug: 0.927

Synth: 0.671

Fig. 4 Training runs with molecules of 20 atoms or less. Results are shown for control (blue) and drug replacement with recombination (green). a Histogram showing number of new molecules produced in control run for different drug-likeness scores. $\mathbf{b}$ Histogram showing number of new molecules produced in our approach using updates to the training data for different drug-likeness scores. $\mathbf{c}$ A few sample new molecules from the drug replacement with recombination run

for the generator trained using drug replacement strategy and recombination. It is illustrative to consider the closest (as measured by Morgan fingerprints [20] and Tanimoto similarity) training set molecule for each example (Additional file 1: Figure S5). For most of the example top performers, only small rearrangements (e.g. changing an atom type or extending a chain) are necessary to provide a boost in the drug-likeness. The prevalence of small rearrangements in the generated molecules, however, is intuitively expected due to constraining the search space to molecules with 9 atoms or less.

The success of guided training data replacement and recombination, as seen in the over 10x improvement over control (see Figs. 1, 2), motivated us to apply our approach to a more realistic data set for drug discovery. Therefore, we extended the training procedure to molecules with 20 or less atoms and added $10 \mathrm{k} \mathrm{mol-}$ ecules from the ZINC [21] rings data set (see "Methods" section). Our results (see Fig. 4) show that our approach again provides a drastic improvement over the traditional GAN for search. The total number of molecules produced increases over the control run (i.e. no replacement, no recombination) by an order of magnitude $\left(\sim 10^{5}\right.$ to $\left.\sim 10^{6}\right)$. Furthermore, the distribution of drug-likeness scores is altered drastically to favor high scoring compounds (Fig. 4a, b).

A sample of some of the top performers are shown in Figure 4c. The closest molecule from the training set for each top performer is shown in Figure S6. Unlike the data constrained to 9 atoms or less, the examples show substantial rearrangements of functional groups compared to the training set. Furthermore, the rearrangements result in a substantial boost in drug-likeness, which reflects the large shift in the histogram for produced molecules (Fig. 4a, b).

To more systematically understand the change in properties for molecules produced using selection and recombination compared to the traditional approach, we computed the distributions for additional metrics (Additional file 1: Figures S7-S11, Table S1). Additional file 1: Figure $S 7$ shows the fraction of each molecule occupied by a given atom type $(\mathrm{C}, \mathrm{N}, \mathrm{O}, \mathrm{F})$. The molecules produced by the drug+re strategy show a shift towards higher $\mathrm{C}, \mathrm{F}$ content and lower $\mathrm{O}, \mathrm{N}$ content compared to the training data and control strategy. Additional file 1: Figure S8 shows the number of atoms, number of rings, and length of rings for the molecules. Again, the distributions for the drug+re strategy show 
a noticeable shift from the training data, with larger molecules, more rings, and larger rings.

An additional 3 metrics (number of rotatable bonds, polar surface area, and Crippen $\operatorname{LogP}[22]$ ) are shown in Additional file 1: Figure S9. It is important to note that these metrics are commonly utilized to filter drug candidates $[18,23]$. Both number of rotatable bonds and Crippen $\log P$ show a substantial increase for the molecules from drug+re compared to the training data. The shifts for these metrics can be anticipated as they are both used to determine the drug-likeness score. Polar surface areas is also used in the drug-likeness score provided by rdkit but with a much smaller weight.

The shift of the drug+re distribution away from the original training data can also be quantified using fingerprint similarity. To determine the distance of produced molecules from the original data, we computed the Morgan fingerprints for each molecule in rdkit. We then found the closest molecule based on Tanimoto similarity (and corresponding distance). Additional file 1: Figure S10 shows the distributions of the minimum distance for both control and drug+re strategies. In agreement with the other metrics, molecules produced by the drug+re strategy on average have a larger minimum distance than the control molecules. Therefore, the drug+re strategy not only produces more molecules than control, it produces more distinct molecules relative to the training set.

As a final comparison, Additional file 1: Figure S11 shows the drug-likeness distributions across different selection and recombination strategies. The drug+re strategy shows a clear shift towards high scoring drug molecules compared to all other options. It is interesting to note that although recombination does provide a clear benefit in producing higher scoring molecules alone (i.e. compare control to control+re), updating the training data through selection generates a substantial shift in the probability density towards high performers.

The difficulty of mode collapse presents a major challenge to researchers using GANs for discovery. Previous attempts to prevent mode collapse have altered the loss function [14, 15], however, the issue has still remained in drug discovery efforts $[11,12]$. Our approach, updating the training data, eliminated the plateau in new molecule discovery compared to the control case without any updates to the minimax loss function. Furthermore, recombination amplified the increase in new molecules for all replacement strategies. Together, these results suggest that replacement and recombination can drastically accelerate the use of GANs for drug discovery.

One limitation of the current approach is that a definition for valid generated samples must be given. In the current context, valid molecules are determined by the ability of rdkit to parse and create the proposed molecule. However, in other contexts, the definition of valid may not be so straightforward (e.g. what defines a valid image). In these cases, some scoring function must be introduced to determine replacement/validity. This highlights the importance of developing useful domain specific metrics for ML applications, including drug discovery [2].

Allowing updates to the training data provides much needed flexibility towards utilizing GANs in drug discovery. This can be seen in our search for drug-like compounds with 20 atoms or less. The initial training set only contained $10 \%$ of molecules with more than 9 atoms (the rest coming from QM9), however, through replacement and recombination, the search adapted to explore regions of parameter space with higher scores. The ability to adapt relieves some of the pressure in generating large data sets for each new desired task, as an incremental approach can be used.

Updates to the training data can be placed within a broader context of data augmentation for GANs. Recent work [24, 25] has explored ways to improve GAN training on images by augmenting labeled data while preserving the underlying target distribution. Data augmentation techniques are particularly relevant due to the inherent costs associated with manual labeling. In the context of drug discovery, our results show that search for novel compounds is broadened by allowing the GAN to explore regions of parameter space outside the original training set through incremental updates. The key tradeoff is that features of the original distribution may be lost as the training data shifts. The type of application and diversity of the training data can then be used to determine the costs and benefits associated with training data updates. In cases where labeled data is abundant and diverse, traditional approaches to training can be used. In cases with limited initial data, or limited initial data with desired characteristics, training updates can be used to improve search performance.

Our approach in updating the training data also has many connections to previous searches over chemical space using genetic algorithms [26-28]. For a genetic algorithm, hand-crafted rules are created for mutation (e.g. switch an atom type, delete an atom, add an atom) and recombination (e.g. swap functional groups between two molecules). Iterations of mutations and recombination are then performed on an initial population, with selection occurring to improve fitness in subsequent populations. In this context, the current work serves as a step towards automating the manual creation of rules for mutation. The generator network serves to produce candidate molecules based on the current training population, which is updated over time. Automating the process 
of recombination (in addition to mutation) is an interesting direction for future work.

Previous work has explored a possible way to incorporate genetic algorithms into GAN training [29]. More specifically, mutation and recombination were applied directly to the generator output in order to stabilize the training of the discriminator. This approach can be broadly categorized with other efforts to promote diversity during GAN training through data augmentation [24, 25 ] in contrast to our approach of updating the training data. It is important to note that improvements to model architecture and approaches to prevent overfitting, such as dropout, would not alleviate the need for updates to the training data in search. Approaches to prevent overfitting, by design, would enable the model to more fully reproduce the distribution underlying the training data, however, they would not promote exploration beyond the training data as needed in search applications.

Many of the advances in training GANs $[14,15]$ should be complementary to our approach. Here, we have utilized a relatively simple architecture, i.e. fully connected networks with a few layers for both generator and discriminator, and the standard GAN loss function. By adding replacement and recombination, however, large gains were seen in both the number and quality (i.e. drug score) of new molecules produced. The addition of more sophisticated networks (e.g. GCN [30]) to scale the current approach to larger molecules is an interesting direction for future investigations.

\section{Conclusions}

Generative machine learning models, including GANs, are a powerful tool towards searching chemical space for desired functionalities. Here, we have presented a strategy for promoting search beyond the original training set using incremental updates to the data. Our approach builds upon the concepts of selection and recombination common in Genetic Algorithms and can be seen as a step towards automating the typically manual rules for mutation. Our results suggest that updates to the data enable a larger number of compounds to be explored, leading to an increase in high performing candidates compared to a fixed training set.

\section{Methods}

\section{Data}

The original training data used for all models was taken from QM9 [17], a subset of the GDB-17 chemical database [31], as reported in a previous study [11]. The data was downloaded from deepchem ${ }^{1}$ and then processed using rdkit [19], with any molecules that caused errors during sanitization removed. Only the first 100k (out of $\sim 133 \mathrm{k}$ ) compounds were then used in training.

To modify the training data to include larger molecules (i.e. up to 20 atoms), a subset of the ZINC [21] rings data set was used. Smiles data was downloaded from ZINC $^{2}$ and filtered to include molecules with between 10 and 20 atoms that contain only $\mathrm{C}, \mathrm{N}$, and $\mathrm{O}$. The first $10 \mathrm{k}$ molecules were then used to replace the first $10 \mathrm{k}$ entries from the original training data. The resulting training data had 100k molecules, with 90k from QM9 [17] and 10k from Zinc [21].

\section{Models}

The GAN was implemented using pytorch [32], with both the discriminator and generator consisting of 4 fully connected layers. The generator received as input normally distributed random vectors with dimension 8 . The output of the generator was an adjacency matrix with the off-diagonal elements specifying the bond order and the on-diagonal elements specifying the atom type. The discriminator received as input the one hot representation of the adjacency matrix and output a single real number. We utilized the standard GAN minimax loss for training [9]. The Adam optimizer[33] was used with a learning rate of $10^{-4}$ for all runs.

\section{Updates to training data}

All models were trained in intervals of 5 epochs. During each epoch $10 \mathrm{k}$ samples were collected from the generator. Samples that were both novel and valid were aggregated over the 5 epochs. Then, a replacement strategy (random or drug) was applied to the original training data. For random replacement, current training samples were randomly selected and replaced. For drug replacement, current samples were sorted in ascending order of drug-likeness score. Updates were only made if the new sample had a greater score than the sample being replaced. Additional metrics (i.e. synthesizability and solubility) used the same update procedure as drug-likeness.

For both replacement strategies, we also considered recombination. In recombination, half of the 10k generator sample was combined with the current training data using crossover. In crossover, a sample is selected from the current training data and copied into a new adjacency matrix. Then, a random integer is uniformly sampled between 1 and the length of the adjacency matrix. The corresponding slice from the generated matrix (e.g. first 5 rows and columns) overwrites the same region of the

\footnotetext{
${ }^{1}$ http://deepchem.io.s3-website-us-west-1.amazonaws.com/datasets/gdb9.tar gz.

${ }^{2}$ https://zinc.docking.org/rings.smi?count=all.
} 
copied matrix to produce a new candidate molecule. For the drug replacement strategy, samples were drawn from the training set weighted by drug score. The weights were determined by taking the softmax of the current metric scores for the training data. Replacement then proceeded as previously stated. Additional metrics (i.e. synthesizability and solubility) used the same update procedure as drug-likeness.

\section{Metrics}

To track the performance of each GAN during training, we relied upon two key metrics: the number of new molecules generated and the quantitative estimation of drug-likeness score $[11,18,19]$. Determining that a molecule is new was accomplished by comparing the canonical smiles representation of the compound with the full training set and any molecules produced up to that point. Generation of a canonical smiles string for a given molecule was performed using rdkit [19]. To show that the results are not unique to drug-likeness, we also included runs for selection of synthesizability and solubility. The metrics calculations were performed as previously reported [11, 12, 18, 34].

Additional metrics for analysis as shown in Additional file 1: Figures S7-S11 were all computed using built-in functionality from rdkit [19]. Similarity (and corresponding distance) between molecules was computed by generating Morgan fingerprints [20] and using Tanimoto similarity as preformed in a previous study [11].

\section{Supplementary Information}

The online version contains supplementary material available at https://doi. org/10.1186/s13321-021-00494-3.

Additional file 1: Supporting Information.: Figures S1-S11 and Table S1.

\section{Acknowledgements}

This research used resources of the Oak Ridge Leadership Computing Facility at the Oak Ridge National Laboratory, which is supported by the Office of Science of the U.S. Department of Energy under Contract No. DE-AC05-00OR22725.

This manuscript has been authored by UT-Battelle LLC under Contract No. DE-AC05-00OR22725 with the US Department of Energy (DOE). The US government retains and the publisher, by accepting the article for publication, acknowledges that the US government retains a nonexclusive, paid-up, irrevocable, worldwide license to publish or reproduce the published form of the manuscript, or allow others to do so, for US government purposes. DOE will provide public access to these results of federally sponsored research in accordance with the DOE Public Access Plan (http://energy.gov/downloads/ doe-public-access-plan)

\section{Authors' contributions}

All authors contributed in developing the concept for the study. AEB and DB developed the code and trained the models. CS performed initial testing of the code. AEB performed data analysis and generated the figures. All authors assisted in manuscript preparation. All authors read and approved the final manuscript.

\section{Funding}

The research was supported by the U.S. Department of Energy, Office of Science, through the Office of Advanced Scientific Computing Research (ASCR), under contract number DE-AC05-00OR22725; the Exascale Computing Project (ECP) (17-SC-20-SC), a collaborative effort of the U.S. Department of Energy Office of Science and the National Nuclear Security Administration; and in part by the Joint Design of Advanced Computing Solutions for Cancer (JDACS4C) program established by the U.S. Department of Energy (DOE) and the National Cancer Institute ( $\mathrm{NCl}$ ) of the National Institutes of Health. It was performed under the auspices of the U.S. Department of Energy by Argonne National Laboratory under Contract DE-AC02-06-CH11357, Lawrence Livermore National Laboratory under Contract DE-AC52-07NA27344, Los Alamos National Laboratory under Contract DE-AC5206NA25396, Oak Ridge National Laboratory under Contract DE-AC05-00OR22725, and Frederick National Laboratory for Cancer Research under Contract HHSN261200800001E.

\section{Availability of data and materials}

The training data used for this study can be downloaded as described in "Methods" section (Data subsection).

\section{Competing interests}

The authors declare that they have no competing interests.

Received: 29 December 2020 Accepted: 9 February 2021

Published online: 23 February 2021

\section{References}

1. Sanchez-Lengeling B, Aspuru-Guzik A (2018) Inverse molecular design using machine learning: generative models for matter engineering. Science 361 (6400):360-365. https://doi.org/10.1126/science.aat2663

2. Vanhaelen Q, Lin Y-C, Zhavoronkov A (2020) The advent of generative chemistry. ACS Med Chem Lett 11(8):1496-1505. https://doi.org/10.1021/ acsmedchemlett.0c00088

3. Joo S, Kim MS, Yang J, Park J (2020) Generative model for proposing drug candidates satisfying anticancer properties using a Cconditional variational autoencoder. ACS Omega 5(30):18642-18650. https://doi. org/10.1021/acsomega.0c01149

4. Maziarka Ł, Pocha A, Kaczmarczyk J, Rataj K, Danel T, Warchoł M (2020) Mol-CycleGAN: A generative model for molecular optimization. J Cheminform 12(1):1-18. https://doi.org/10.1186/s13321-019-0404-1

5. Zhavoronkov A, Ivanenkov YA, Aliper A, Veselov MS, Aladinskiy VA, Aladinskaya AV, Terentiev VA, Polykovskiy DA, Kuznetsov MD, Asadulaev A, Volkov Y, Zholus A, Shayakhmetov RR, Zhebrak A, Minaeva LI, Zagribelnyy BA, Lee LH, Soll R, Madge D, Xing L (2019) Deep learning enables rapid identification of potent DDR1 kinase inhibitors. Nature Biotechnology. https://doi.org/10.1038/s41587-019-0224-x

6. Bhowmik D, Gao S, Young MT, Ramanathan A (2018) Deep clustering of protein folding simulations. BMC Bioinform 19(18):47-58

7. Romero R, Ramanathan A, Yuen T, Bhowmik D, Mathew M, Munshi LB, Javaid S, Bloch M, Lizneva D, Rahimova A, Khan A, Taneja C, Kim S-M, Sun L, New M, Haider S, Zaidi M (2019) Mechanism of glucocerebrosidase activation and dysfunction in gaucher disease unraveled by molecular dynamics and deep learning. Proc Natl Acad Sci 116(11):5086-5095

8. Chen H, Engkvist O, Wang Y, Olivecrona M, Blaschke T (2018) The rise of deep learning in drug discovery. Drug Discov Today 23(6):1241-1250. https://doi.org/10.1016/j.drudis.2018.01.039

9. Goodfellow I, Pouget-Abadie J, Mirza M, Xu B, Warde-Farley D, Ozair S, Courville A, Bengio Y (2014) Generative adversarial nets. In: Ghahramani, Z., Welling, M., Cortes, C., Lawrence, N.D., Weinberger, K.Q. (eds.) Advances in Neural Information Processing Systems 27, vol. 2014-December, pp. 2672-2680. http://papers.nips.cc/paper/5423-generative-adversaria I-nets.pdf

10. Goodfellow I (2017) NIPS 2016 Tutorial: Generative Adversarial Networks. arxiv:1701.00160

11. De Cao N, KipfT (2018) MolGAN: An implicit generative model for small molecular graphs. ICML 2018 workshop on Theoretical Foundations and Applications of Deep Generative Models 
12. Guimaraes GL, Sanchez-Lengeling B, Outeiral C, Farias PLC, Aspuru-Guzik A (2017) Objective-Reinforced Generative Adversarial Networks (ORGAN) for Sequence Generation Models. arxiv:1705.10843

13. Benhenda M (2017) ChemGAN challenge for drug discovery: can Al reproduce natural chemical diversity?. arxiv:1708.08227

14. Arjovsky M, Chintala S, Bottou L (2017) Wasserstein GAN. arxiv:1701.07875

15. Gulrajani I, Ahmed F, Arjovsky M, Dumoulin V, Courville A (2017) Improved Training of Wasserstein GANs. In: Advances in Neural Information Processing Systems 31, vol. 2017-December, pp. 5768-5778. arxiv:1704.00028

16. Eiben $A E$, Smith JE (2015) Introduction to evolutionary computing, 2nd edn. Springer, Springer-Verlag GmbH Germany

17. Ramakrishnan R, Dral PO, Rupp M, Von Lilienfeld OA (2014) Quantum chemistry structures and properties of 134 kilo molecules. Sci Data 1:1-7. https://doi.org/10.1038/sdata.2014.22

18. Bickerton GR, Paolini GV, Besnard J, Muresan S, Hopkins AL (2012) Quantifying the chemical beauty of drugs. Nat Chem 4(2):90-98. https://doi. org/10.1038/nchem.1243

19. RDKit: Open-source cheminformatics. http://www.rdkit.org

20. Rogers D, Hahn M (2010) Extended-connectivity fingerprints. J Chem Inf Model 50(5):742-754. https://doi.org/10.1021/ci100050t

21. Sterling T, Irwin JJ (2015) ZINC 15 - Ligand discovery for everyone. J Chem Inf Model 55(11):2324-2337. https://doi.org/10.1021/acs.jcim.5b00559

22. Wildman SA, Crippen GM (1999) Prediction of physicochemical parameters by atomic contributions. J Chem Inf Comput Sci 39(5):868-873. https://doi.org/10.1021/ci9903071

23. Veber DF, Johnson SR, Cheng HY, Smith BR, Ward KW, Kopple KD (2002) Molecular properties that influence the oral bioavailability of drug candidates. J Med Chem 45(12):2615-2623. https://doi.org/10.1021/jm020 $017 n$

24. Zhao S, Liu Z, Lin J, Zhu J-Y, Han S (2020) Differentiable Augmentation for Data-Efficient GAN Training. arxiv:2006.10738

25. Zhao Z, Zhang Z, Chen T, Singh S, Zhang H (2020) Image Augmentations for GAN Training. arxiv:2006.02595

26. Virshup AM, Contreras-García J, Wipf P, Yang W, Beratan DN (2013) Stochastic voyages into uncharted chemical space produce a representative library of all possible drug-like compounds. J Am Chem Soc 135(19):7296-7303. https://doi.org/10.1021/ja401184g

27. Jensen JH (2019) A graph-based genetic algorithm and generative model/Monte Carlo tree search for the exploration of chemical space. Chem Sci 10(12):3567-3572. https://doi.org/10.1039/c8sc05372c

28. Brown N, McKay B, Gilardoni F, Gasteiger J (2004) A graph-based genetic algorithm and its application to the multiobjective evolution of median molecules. J Chem Inf Comput Sci 44(3):1079-1087. https://doi. org/10.1021/ci034290p

29. Cho H.Y, Kim Y.H (2019) Stabilized training of generative adversarial networks by a genetic algorithm. GECCO 2019 Companion - Proceedings of the 2019 Genetic and Evolutionary Computation Conference Companion 1:51-52. https://doi.org/10.1145/3319619.3326774

30. Sun M, Zhao S, Gilvary C, Elemento O, Zhou J, Wang F (2020) Graph convolutional networks for computational drug development and discovery. Brief Bioinform 21(3):919-935. https://doi.org/10.1093/bib/bbz042

31. Ruddigkeit L, Van Deursen R, Blum LC, Reymond JL (2012) Enumeration of 166 billion organic small molecules in the chemical universe database GDB-17. J Chem Inf Model 52(11):2864-2875. https://doi.org/10.1021/ ci300415d

32. Paszke A, Gross S, Chintala S, Chanan G, Yang E, DeVito Z, Lin Z, Desmaison A, Antiga L, Lerer A (2017) Automatic differentiation in pytorch. In: NIPS-W

33. Kingma DP, Ba J (2017) Adam: A method for stochastic optimization. arxiv :1412.6980

34. Ertl P, Schuffenhauer A (2009) Estimation of synthetic accessibility score of drug-like molecules based on molecular complexity and fragment contributions. J Cheminf 1(1):1-11. https://doi.org/10.1186/1758-2946-1-8

\section{Publisher's Note}

Springer Nature remains neutral with regard to jurisdictional claims in published maps and institutional affiliations.
Ready to submit your research? Choose BMC and benefit from:

- fast, convenient online submission

- thorough peer review by experienced researchers in your field

- rapid publication on acceptance

- support for research data, including large and complex data types

- gold Open Access which fosters wider collaboration and increased citations

- maximum visibility for your research: over $100 \mathrm{M}$ website views per year

At $\mathrm{BMC}$, research is always in progress.

Learn more biomedcentral.com/submissions 